\title{
ОБ УТОЧНЕНИИ ОЦЕНОК ПОКАЗАТЕЛЕЙ ЛЯПУНОВА ОДНОГО КЛАССА ЛИНЕЙНЫХ НЕАВТОНОМНЫХ СИСТЕМ РАЗНОСТНЫХ УРАВНЕНИЙ
}

\author{
А. В. Ласунский
}

Получена оценка нормы квадратной матрицы $A^{t}$ порядка $n$ :

$$
\left\|A^{t}\right\| \leq \sum_{k=0}^{n-1} C_{t}^{k} \gamma^{t-k}(\gamma+\|A\|)^{k}, \quad t \geq n-1,
$$

где $C_{t}^{k}-$ биномиальный коэффициент; $\gamma=\max _{i}\left|\lambda_{i}\right| ; \lambda_{i}-$ собственные числа матрицы $A$. С помощью этой оценки методом замораживания получены уточнения констант в оценке сверху для старшего $\Lambda$ и в оценке снизу для младшего $\lambda$ показателей системы $x(t+1)=A(t) x(t), x \in \mathbb{R}^{n}, t \in \mathbb{Z}^{+}$с вполне ограниченной матрицей $A(t)$. Предполагается, что матрицы $A(t)$ и $A^{-1}(t)$ для любых $t, s \in \mathbb{Z}^{+}$удовлетворяют неравенствам $\|A(t)-A(s)\| \leq \delta|t-s|^{\alpha},\left\|A^{-1}(t)-A^{-1}(s)\right\| \leq \delta|t-s|^{\alpha}$ с некоторыми постоянными $0<\alpha \leq 1$ и $\delta>0$. На примере показано, что постоянные $\gamma$ и $\delta$, вообще говоря, связаны между собой.

Ключевые слова: оценки показателей Ляпунова, метод замораживания для дискретных систем.

A. V. Lasunskii. Refinement of estimates for the Lyapunov exponents of a class of linear nonautonomous systems of difference equations.

We obtain an estimate for the norm of an $n$ th-order square matrix $A^{t}$ :

$$
\left\|A^{t}\right\| \leq \sum_{k=0}^{n-1} C_{t}^{k} \gamma^{t-k}(\gamma+\|A\|)^{k}, \quad t \geq n-1,
$$

where $C_{t}^{k}$ are the binomial coefficients, $\gamma=\max _{i}\left|\lambda_{i}\right|$, and $\lambda_{i}$ are the eigenvalues of $A$. Based on this estimate and using the freezing method, we improve the constants in the upper and lower estimates for the highest and lowest exponents, respectively, of the system $x(t+1)=A(t) x(t), x \in \mathbb{R}^{n}, t \in \mathbb{Z}^{+}$, with a completely bounded matrix $A(t)$. It is assumed that the matrices $A(t)$ and $A^{-1}(t)$ satisfy the inequalities $\|A(t)-A(s)\| \leq$ $\delta|t-s|^{\alpha},\left\|A^{-1}(t)-A^{-1}(s)\right\| \leq \delta|t-s|^{\alpha}$ with some constants $0<\alpha \leq 1$ and $\delta>0$ for any $t, s \in \mathbb{Z}^{+}$. We give an example showing that the constants $\gamma$ and $\delta$ are generally related.

Keywords: estimates for Lyapunov exponents, freezing method for discrete systems.

MSC: 39A30, 39A22

DOI: $10.21538 / 0134-4889-2020-26-3-84-90$

\section{1. Введение}

Линейные неавтономные системы разностных уравнений представляют интерес и как самостоятельный объект, и как вспомогательный при исследовании свойств решений нелинейных систем методом линеаризации. В [1] приведена обширная библиография по этому вопросу. У авторов этой статьи большой цикл публикаций, посвященных дискретным системам. Хотим отметить работы [2-4], подчеркивающие актуальность рассматриваемой задачи. Вопросы устойчивости решений дискретных систем получили всестороннее развитие и достигли уровня, сравнимого с теорией устойчивости решений систем дифференциальных уравнений. Здесь уместно сказать, что в последние годы активно разрабатывается теория характеристик колеблемости и блуждаемости решений линейной дифференциальной системы. Первый шаг в этом направлении сделал И. Н. Сергеев, введя определение характеристической частоты скалярной функции [5]. С публикациями по этой тематике можно также можно ознакомиться в его статье [6]. По-видимому, этот вопрос для дискретных систем еще не разрабатывался. 
В работе [7] для старшего $\Lambda$ и младшего $\lambda$ показателей системы

$$
x(t+1)=A(t) x(t), \quad\|A(t)\| \leq M, \quad\left\|A^{-1}(t)\right\| \leq M, \quad x \in \mathbb{R}^{n}, \quad t \in \mathbb{Z}^{+},
$$

при условии, что матрицы $A(t)$ и $A^{-1}(t)$ для любых $t, s \in \mathbb{Z}^{+}$удовлетворяют неравенствам

$$
\begin{gathered}
\|A(t)-A(s)\| \leq \delta|t-s|^{\alpha}, \\
\left\|A^{-1}(t)-A^{-1}(s)\right\| \leq \delta|t-s|^{\alpha}
\end{gathered}
$$

с некоторыми постоянными $0<\alpha \leq 1$ и $\delta>0$, получены оценки

$$
\Lambda \leq \ln \gamma+C \delta^{1 /(n+\alpha)}, \quad \lambda \geq \ln \omega-C \delta^{1 /(n+\alpha)} .
$$

Здесь

$$
\gamma=\sup _{t \in \mathbb{Z}^{+}} \max _{i}\left|\lambda_{i}(t)\right|, \quad \omega=\inf _{t \in \mathbb{Z}^{+}} \min _{i}\left|\lambda_{i}(t)\right|>0,
$$

$\lambda_{i}(t), i=\overline{1, n},-$ собственные числа матрицы $A(t)$ системы (l), $C=L \gamma^{-1}+n+\alpha-1$, постоянная $L$ из оценки

$$
\left\|A^{t}\left(t_{1}\right)\right\| \leq L(1+t)^{n-1} \gamma^{t}, \quad t_{1}, t \in \mathbb{Z}^{+} .
$$

В публикациях $[8 ; 9]$ изучался случай $\alpha=1$ при условии

$$
\|A(t+1)-A(t)\| \leq \delta .
$$

Заметим, что неравенство (2) с $\alpha=1$ и неравенство (5) равносильны. Действительно, пусть выполнено неравенство (5), тогда из неравенства треугольника для нормы имеем для $t>s$

$$
\|A(t)-A(s)\| \leq \sum_{i=s}^{t-1}\|A(i+1)-A(i)\| \leq \delta(t-s) .
$$

Обратное очевидно.

В настоящей работе мы уточним постоянную $C$ в оценках (3). Заметим, что постоянные $\gamma$ и $\delta$ для матрицы $A(t)$, вообще говоря, связаны между собой. Утверждать, что если $0<\gamma<1$, то при достаточно малом $\delta>0$ значение $\ln \gamma+C \delta^{1 /(n+\alpha)}<0$, нельзя.

П р и м е р. Система (1) с матрицей

$$
A(t)=\left(\begin{array}{cc}
a \exp ((t+2) \sin \ln (t+2)-(t+1) \sin \ln (t+1)) & 0 \\
1 & \exp (-|a|)
\end{array}\right),
$$

$a \neq 0$, неправильна, так как не существует строгий предел

$$
\lim _{t \rightarrow+\infty} \frac{1}{t} \ln \left|\prod_{i=0}^{t-1} a_{11}(i)\right|=\ln |a|+\lim _{t \rightarrow+\infty} \frac{t+1}{t} \sin \ln (t+1) .
$$

Из теоремы Лагранжа для функции $\varphi(t)=(t+1) \sin \ln (t+1), t \in[0 ;+\infty)$, следует, что $|\varphi(t+1)-\varphi(t)| \leq \sqrt{2}$. Значит, матрица $A(t)$ вполне ограничена (ограничена вместе с обратной). Так как у треугольной матрицы собственные числа стоят на главной диагонали, то $\gamma=\max (|a| \exp (\sqrt{2}) ; \exp (-|a|))=\exp (-|a|)<1$ при достаточно малых по модулю значениях параметра $a$.

Имеем

$$
\left|a_{11}(t)-a_{11}(s)\right|=\left|a_{11}^{\prime}(c)\right||t-s| \leq(|a| 2 \sqrt{2} \exp \sqrt{2})|t-s|=\delta|t-s| .
$$

Неравенство (2) выполняется с $\alpha=1$, причем положительную постоянную $\delta$ можно сделать сколь угодно малой за счет выбора параметра $a$. Величина $\ln \gamma+C \delta^{1 /(n+\alpha)}=\ln \gamma+C \delta^{1 / 3}=$ $-|a|+C_{1}|a|^{1 / 3}>0$, если значение $|a|$ близко к нулю. 


\section{2. Оценка нормы матрицы $A^{t}$}

В следующей лемме мы получим оценку нормы матрицы $A^{t}$. Эту оценку мы будем использовать в дальнейшем.

Лемма. Пусть $\lambda_{i}-$ собственные числа матрицы $A$ порлдка $n$ u $\gamma=\max _{i}\left|\lambda_{i}\right|$, тогда $\partial л я$ всех натуральных $t \geq n-1$ справедлива оченка

$$
\left\|A^{t}\right\| \leq \sum_{k=0}^{n-1} C_{t}^{k} \gamma^{t-k}(\gamma+\|A\|)^{k}
$$

где $C_{t}^{k}-$ биномиалъный коэффициент.

Д о к а з а т е л ь с т в о. Воспользуемся идеей доказательства оценки матричной экспоненты $\exp (A t)[10$, с. $78 ; 11$, с. $131 ; 12$, с. 163$]$.

Для любой аналитической функции $f(z)$ и матрицы $A$-го порядка имеет место равенство

$$
f(A)=b_{1} E+b_{2}\left(A-\lambda_{1} E\right)+b_{3}\left(A-\lambda_{1} E\right)\left(A-\lambda_{2} E\right)+\ldots+b_{n}\left(A-\lambda_{1} E\right) \ldots\left(A-\lambda_{n-1} E\right),
$$

в котором $E-$ единичная матрица, $b_{1}=f\left(\lambda_{1}\right)$,

$$
b_{k+1}=\int_{0}^{t_{0}} d t_{1} \ldots \int_{0}^{t_{k-1}} f^{(k)}\left(\lambda_{1}+\left(\lambda_{2}-\lambda_{1}\right) t_{1}+\ldots+\left(\lambda_{k+1}-\lambda_{k}\right) t_{k}\right) d t_{k}, \quad k=1, \ldots, n-1 .
$$

При желании можно считать, что формула для коэффициента $b_{1}$ получается из общей формулы (8) при $k=0$ и очевидных допущениях. Переменные интегрирования изменяются в пределах $0 \leq t_{k} \leq \ldots \leq t_{1} \leq t_{0}=1$.

Имеем

$$
\left.\lambda_{1}+\left(\lambda_{2}-\lambda_{1}\right)\right) t_{1}+\ldots+\left(\lambda_{k+1}-\lambda_{k}\right) t_{k}=\lambda_{1}\left(t_{0}-t_{1}\right)+\lambda_{2}\left(t_{1}-t_{2}\right)+\ldots+\lambda_{k+1}\left(t_{k}-t_{k+1}\right) .
$$

Мы положили $t_{k+1}=0$ и учли, что $t_{0}=1$.

Из формулы (7), получаем

$$
\|f(A)\| \leq\left|b_{1}\right|+\sum_{k=1}^{n-1}\left|b_{k+1}\right|\left(\|A\|+\left|\lambda_{1}\right|\right) \ldots\left(\|A\|+\left|\lambda_{k}\right|\right) \leq\left|b_{1}\right|+\sum_{k=1}^{n-1}\left|b_{k+1}\right|(\|A+\gamma\|)^{k} .
$$

Оценим модули коэффициентов $b_{i}, i=1, \ldots, n$.

Для функции $f(z)=z^{t}$ имеем $f^{(k)}(z)=t(t-1) \ldots(t-k+1) z^{t-k}$, поэтому для $t \geq n-1$ и $k=1,2, \ldots, n-1$ с учетом формулы (9) выводим

$$
\begin{gathered}
\left.\left|f\left(\lambda_{1}\right)\right| \leq \gamma^{t}, \quad \mid f^{(k)}\left(\lambda_{1}+\left(\lambda_{2}-\lambda_{1}\right)\right) t_{1}+\ldots+\left(\lambda_{k+1}-\lambda_{k}\right) t_{k}\right) \mid \\
=t(t-1) \ldots(t-k+1)\left|\sum_{i=0}^{k}\left(t_{i}-t_{i+1}\right) \lambda_{i+1}\right|^{t-k} \leq t(t-1) \ldots(t-k+1)\left(\gamma \sum_{i=0}^{k}\left(t_{i}-t_{i+1}\right)\right)^{t-k} \\
=t(t-1) \ldots(t-k+1) \gamma^{t-k} .
\end{gathered}
$$

Мы учли, что $\sum_{i=0}^{k}\left(t_{i}-t_{i+1}\right)=1$ и $t-k \geq 0$. Далее из формул (8) следует, что

$$
\left|b_{1}\right| \leq \gamma^{t} ; \quad\left|b_{k+1}\right| \leq t(t-1) \ldots(t-k+1) \gamma^{t-k} \int_{0}^{t_{0}} d t_{1} \ldots \int_{0}^{t_{k-1}} d t_{k}=C_{t}^{k} \gamma^{t-k} .
$$

Неравенство (6) леммы доказано. 
3 а м е ч а н и е 1. В дальнейшем нас будет интересовать оценка (6) для случая $\gamma>0$, но лемма справедлива, разумеется, и при $\gamma=0$. Действительно, пусть все собственные числа матрицы $A$ порядка $n$ равны 0, тогда $\gamma=0$. Если $t=n-1$, то оценка (6) превращается в неравенство $\left\|A^{n-1}\right\| \leq\|A\|^{n-1}$. Если $t \geq n$, то неравенство (6) дает $\left\|A^{t}\right\|=0$, что согласуется с тем, что $A^{t}=\mathbb{O}$ для всех $t \geq n$. Действительно, пусть $S^{-1} A S=B=\operatorname{diag}\left[B_{1}, \ldots, B_{p}\right]-$ жорданова форма матрицы А, тогда $A^{t}=S B^{t} S^{-1}=S \operatorname{diag}\left[B_{1}^{t} ; \ldots ; B_{p}^{t}\right] S^{-1}$. Клетка Жордана порядка $k$, соответствующая собственному числу 0 , является нильпотентной матрицей порядка $k$, поэтому, по крайней мере начиная со степени $n$, все степени матрицы являются нулевыми матрицами.

\section{3. Оценки показателей Ляпунова}

В этом разделе с помощью предыдущей леммы методом замораживания мы получим оценку сверху для старшего и оценку снизу для младшего показателя Ляпунова дискретной системы (1).

Теорема. Для старшего $\Lambda$ и младшего $\lambda$ показателей системы (1) справедливы оченки

$$
\begin{gathered}
\Lambda \leq \ln \gamma+\left(\frac{(\gamma+M)^{n-1}}{\gamma^{n}(n-1) !}+n+\alpha-1\right) \delta^{1 /(n+\alpha)}, \\
\lambda \geq \ln \omega-\left(\frac{\left(\omega^{-1}+M\right)^{n-1} \omega^{n}}{(n-1) !}+n+\alpha-1\right) \delta^{1 /(n+\alpha)} .
\end{gathered}
$$

3 а м е ч а н и е 2 . У матриц $A(t)$ и $A^{-1}(t)$ константы $M, \delta, \alpha$ в неравенствах $(1),(2)$ мы считаем общими. Если мы будем считать, что $\left\|A^{-1}(t)\right\| \leq M_{1},\left\|A^{-1}(t)-A^{-1}(s)\right\| \leq \delta_{1}|t-s|^{\alpha_{1}}$, то соответствующим образом изменится оценка для $\lambda$ в формулировке предыдущей теоремы.

Д о к а з а т е л ь с т в о. Зафиксируем $t_{1} \geq n-1$ и перепишем систему (1) в виде

$$
x(t+1)=A\left(t_{1}\right) x(t)+\left(A(t)-A\left(t_{1}\right) x(t) .\right.
$$

По методу вариации произвольных постоянных имеем

$$
x(t)=A^{t}\left(t_{1}\right) x(0)+\sum_{p=0}^{t-1} A^{t-p-1}\left(t_{1}\right)\left(A(p)-A\left(t_{1}\right) x(p),\right.
$$

откуда

$$
\|x(t)\| \leq\left\|A^{t}\left(t_{1}\right)\right\| \cdot\|x(0)\|+\sum_{p=0}^{t-1}\left\|A^{t-p-1}\left(t_{1}\right)\right\| \cdot \|\left(A(p)-A\left(t_{1}\right)\|\cdot\| x(p) \| .\right.
$$

Так как $\|A(t)\| \leq M$ и $\gamma=\sup _{t \in \mathbb{Z}^{+}} \max _{i}\left|\lambda_{i}(t)\right|>0$, то из леммы следует, что

$$
\left\|A^{t}\left(t_{1}\right)\right\| \leq \sum_{k=0}^{n-1} C_{t}^{k} \gamma^{t-k}(\gamma+M)^{k}=\gamma^{t} \varphi(t)
$$

Заметим, что $\varphi(t)$ - многочлен переменной $t$ степени $n-1$ со старшим коэффициентом $(\gamma+M)^{n-1}\left((n-1) ! \gamma^{n-1}\right)^{-1}$. Учитывая неравенство $(2)$, выводим неравенство

$$
\|x(t)\| \leq \gamma^{t} \varphi(t)\|x(0)\|+\sum_{p=0}^{t-1} \gamma^{t-p-1} \varphi(t-p-1) \delta\left|t_{1}-p\right|^{\alpha}\|x(p)\| .
$$


Последнее неравенство справедливо при всех $t \geq n-1$, в том числе и при $t=t_{1} \geq n-1$. Положим $t=t_{1}$, а затем переобозначим $t_{1}$ через $t$, получим

$$
\|x(t)\| \leq \gamma^{t} \varphi(t)\|x(0)\|+\sum_{p=0}^{t-1} \gamma^{t-p-1} \varphi(t-p-1) \delta(t-p)^{\alpha}\|x(p)\|, \quad t \geq n-1 .
$$

Отсюда имеем

$$
\frac{\|x(t)\|}{\gamma^{t} \varphi(t)} \leq\|x(0)\|+\sum_{p=o}^{t-1} \frac{\|x(p)\|}{\gamma^{p+1} \varphi(p)} \frac{\varphi(p) \varphi(t-p-1) \delta(t-p)^{\alpha}}{\varphi(t)} .
$$

Так как $\varphi(p) / \varphi(t) \leq 1$ для $p \leq t-1$ в силу возрастания функции $\varphi(t)$, то приходим к неравенству

$$
\frac{\|x(t)\|}{\gamma^{t} \varphi(t)} \leq\|x(0)\|+\sum_{p=o}^{t-1} \frac{\|x(p)\|}{\gamma^{p+1} \varphi(p)} \cdot \varphi(t-p-1) \delta(t-p)^{\alpha} .
$$

Далее получаем

$$
\begin{gathered}
\varphi(t-p-1)(t-p)^{\alpha} \delta=\varphi(t-p-1)(t-p)^{1-n} \cdot(t-p)^{n+\alpha-1} \delta^{(n+\alpha-1) /(n+\alpha)} \delta^{1 /(n+\alpha)} \\
\left.\leq(\tilde{L}+\varepsilon) \delta^{1 /(n+\alpha)}\left((t-p) \delta^{1 /(n+\alpha)}\right)^{n+\alpha-1} \leq(\tilde{L}+\varepsilon) \delta^{1 /(n+\alpha)} \exp ((n+\alpha-1)(t-p)) \delta^{1 /(n+\alpha)}\right)
\end{gathered}
$$

для всех достаточно больших $t, \varepsilon>0$ сколь угодно мало.

Мы воспользовались неравенством $x^{m}<\exp (m x)$ для $m>0, x>0$. Также мы учли, что $\varphi(t)$ - многочлен переменной $t$ степени $n-1$, поэтому

$$
\lim _{t \rightarrow+\infty} \frac{\varphi(t-p-1)}{(t-p)^{n-1}}=\lim _{t \rightarrow+\infty} \frac{\varphi(t)}{(t+1)^{n-1}}=\frac{(\gamma+M)^{n-1}}{\gamma^{n-1}(n-1) !}=\tilde{L} .
$$

Неравенство (10) преобразуется к виду

$$
\psi(t) \leq\|x(0)\|+\sum_{p=o}^{t-1} \gamma^{-1} \psi(p)(\tilde{L}+\varepsilon) \delta^{1 /(n+\alpha)} .
$$

Мы ввели обозначение $\psi(t)=\|x(t)\|\left(\gamma^{t} \varphi(t) \exp \left((n+\alpha-1) t \delta^{1 /(n+\alpha)}\right)\right)^{-1}$. Кроме того, множитель $\exp \left(-(n+\alpha-1) t \delta^{1 /(n+\alpha)}\right)$, стоящий в правой части неравенства перед $\|x(0)\|$, мы оценили сверху единицей. Применяя дискретный аналог неравенства Гронуолла - Беллмана, получаем

$$
\psi(t) \leq\|x(0)\| \exp \left(\sum_{k=0}^{t-1} \gamma^{-1}(\tilde{L}+\varepsilon) \delta^{1 /(n+\alpha)}\right)=\|x(0)\| \exp \left(t \gamma^{-1}(\tilde{L}+\varepsilon) \delta^{1 /(n+\alpha)}\right) .
$$

Функция $\varphi(t)$ имеет строгий нулевой показатель, поэтому

$$
\chi[x(t)] \leq \ln \gamma+\left(\gamma^{-1} \tilde{L}+n+\alpha-1\right) \delta^{1 /(n+\alpha)},
$$

так как $\varepsilon>0$ произвольно мало.

Получили оценку сверху для старшего показателя $\Lambda$ системы (1). Оценку снизу для младшего показателя $\lambda$ системы (1) получим, перейдя к сопряженной системе

$$
y(t)=\left(A^{-1}(t)\right)^{T} y(t) .
$$


Собственные числа $\mu_{i}(t)$ матрицы $\left(A^{-1}(t)\right)^{T}$ связаны с собственными числами $\lambda_{i}(t)$ матрицы $A(t)$ соотношением $\mu_{i}(t)=\left(\lambda_{i}(t)\right)^{-1}$, поэтому

$$
\sup _{t \in \mathbb{Z}^{+}} \max _{i}\left|\mu_{i}(t)\right|=\sup _{t \in \mathbb{Z}^{+}} \max _{i}\left(\left|\lambda_{i}(t)\right|\right)^{-1}=\left(\inf _{t \in \mathbb{Z}^{+}} \min _{i}\left|\lambda_{i}(t)\right|\right)^{-1}=\omega^{-1} .
$$

Для решений системы (12) имеем оценку, аналогичную оценке (11) с заменой $\gamma$ на $\omega^{-1}$. Из этой оценки следует, что

$$
\chi[y(t)] \leq-\ln \omega+\left(\frac{\left(\omega^{-1}+M\right)^{n-1} \omega^{n}}{(n-1) !}+n+\alpha-1\right) \delta^{1 /(n+\alpha)} .
$$

Для любого решения $x(t) \neq 0$ системы (1) найдется решение $y(t)$ сопряженной системы $(12)$ такое, что $(x(t), y(t))=(x(0), y(0)) \neq 0$.

Так как $(x, y) \leq\|x\| \cdot\|y\|$, то

$$
\chi[x(t)] \geq-\chi[y(t)] \geq \ln \omega-\left(\frac{\left(\omega^{-1}+M\right)^{n-1} \omega^{n}}{(n-1) !}+n+\alpha-1\right) \delta^{1 /(n+\alpha)} .
$$

Получили оценку снизу младшего показателя $\lambda$ системы (1).

Теорема доказана.

З а м е ч а н и е 3. Подборку контпримеров для дискретных линейных систем, показывающих отсутствие связи между поведением решений и собственными числами матрицы коэффициентов системы, можно найти, например, в [13].

\section{СПИСОК ЛИТЕРАТУРЫ}

1. Kuznetsov N.V., Alexeeva T.A., Leonov G.A. Invariance of Lyapunov exponents and Lyapunov dimension for regular and irregular linearizations // Nonlinear Dyn. 2016. Vol. 85. P. 195-201. doi: 10.1007/s11071-016-2678-4 .

2. Czornik A., Nawrat A. On new estimates for Lyapunov exponents of discrete time varying linear systems // Automatica. 2010. Vol. 46, no. 4. P. 775-778. doi: 10.1016/j.automatica.2010.01.014.

3. Czornik A., Mokry P., Nawrat A. On the sigma exponent of discrete linear systems // IEEE Transactions on Automatic Control. 2010. Vol. 55, no. 6. P. 1511-1515. doi: 10.1109/TAC.2010.2045699.

4. Czornik A., Nawrat A., Niezabitowski M. On the Lyapunov exponents of a class of second-order discrete time linear systems with bounded perturbations // Dynamical Systems. 2013. Vol. 28, no. 4. P. 473-483. doi: 10.1080/14689367.2012.748718.

5. Сергеев И.Н. Определение характеристических частот линейного уравнения // Дифференц. уравнения. 2004. Т. 40, № 11. С. 1573.

6. Сергеев И.Н. Характеристики колеблемости и блуждаемости решений линейной дифференциальной системы // Изв. РАН. Серия математическая. 2012. Т. 6, № 1. С. 149-172. doi: $10.4213 / \mathrm{im} 5035$.

7. Ласунский А.В. Оценки решений линейных и квазилинейных систем в неавтономном случае // Дифференц. уравнения. 2016. Т. 52, № 2. С. 177-185.

8. Замковая Л.Д. К методу замораживания для дискретных систем // Дифференц. уравнения. 1980. Т. 16, № 4. С. 697-704.

9. Замковая Л.Д. Оценки показателей экспоненциального роста решений некоторых систем // Дифференц. уравнения. 1988. Т. 24, № 11. С. 2008-2010.

10. Гельфанд И.М., Шилов Г.Е. Некоторые вопросы теории дифференциальных уравнений. Москва: Физматгиз, 1958. 274 с.

11. Былов Б.Ф., Виноград Р.Э., Гробман Д.М., Немыцкий В. В. Теория показателей Ляпунова и ее приложения к вопросам устойчивости. Москва: Наука, 1966. 576 с.

12. Изобов Н.А. Введение в теорию показателей Ляпунова. Минск: БГУ, 2006. 319 с.

13. Ласунский А.В. Устойчивость и собственные числа линейных неавтономных систем разностных и дифференциальных уравнений // Математика в высшем образовании. 2010. № 8. С. 37-40. 
Поступила 28.04.2020

После доработки 16.05.2020

Принята к публикации 30.06.2020

Ласунский Александр Васильевич

д-р физ.-мат. наук, доцент

профессор кафедры прикладной математики и информатики

Новгородский государственный университет имени Ярослава Мудрого

г. Великий Новгород

e-mail: Alexandr.Lasunsky@novsu.ru

\section{REFERENCES}

1. Kuznetsov N.V., Alexeeva T.A., Leonov G.A. Invariance of Lyapunov exponents and Lyapunov dimension for regular and irregular linearizations. Nonlinear Dyn., 2016, vol. 85, pp. 195-201. doi: $10.1007 /$ s11071-016-2678-4.

2. Czornik A., Nawrat A. On new estimates for Lyapunov exponents of discrete time varying linear systems. Automatica, 2010, vol. 46, no. 4, pp. 775-778 . doi: 10.1016/j.automatica.2010.01.014.

3. Czornik A., Mokry P., Nawrat A. On the sigma exponent of discrete linear systems. IEEE Transactions on Automatic Control, 2010, vol. 55, no. 6, pp. 1511-1515 . doi: 10.1109/TAC.2010.2045699.

4. Czornik A., Nawrat A., Niezabitowski M. On the Lyapunov exponents of a class of second-order discrete time linear systems with bounded perturbations. Dynamical Systems, 2013, vol. 28, no. 4, pp. $473-483$. doi: $10.1080 / 14689367.2012 .748718$.

5. Sergeev I.N. Definition of characteristic frequencies of a linear equation. Diff. Eq., 2004, vol. 40, no. 11, p. 1573 (in Russian).

6. Sergeev I.N. Oscillation and wandering characteristics of solutions of a linear differential system.Izvestiya: Mathematics, 2012, vol.76, no.1, pp. 139-162. doi: 10.1070/IM2012v076n01ABEH002578.

7. Lasunskii A.V. Estimates for solutions of Linear and quasilinear systems in the nonautonomous case. Diff. Eq., 2016, vol. 52, no. 2, pp. 177-185. doi: 10.1134/S001226611602004X.

8. Zamkovaya L.D. On a method of "Freezing" for discrete systems. Differ. Uravn., 1980, vol. 16, no. 4, pp. 697-704 (in Russian).

9. Zamkovaya L.D. Estimates of exponents of exponential growth of solutions of some systems. Differ. Uravn., 1988, vol. 24, no. 11, pp. 2008-2010 (in Russian).

10. Gel'fand I.M., Shilov G.E. Generalized functions, vol. 3: Theory of differential equations. Providence: AMS Chelsea Publ., 1967, 222 p. ISBN: 978-1-4704-2661-3. Original Russian text published in Gel'fand I.M., Shilov G.E. Nekotorye voprosy teorii differentsial'nykh uravnenii. Moscow: Fizmatgiz Publ., 1958, 274 p.

11. Bylov B.F., Vinograd R.E., Grobman D.M., and Nemytskii V.V. Teoriya pokazatelei Lyapunova $i$ ee prilozheniya $k$ voprosam ustoichivosti [Theory of Lyapunov exponents and its application to problems of stability]. Moscow: Nauka Publ., 1966, 576 p.

12. Izobov N.A. Vvedenie $v$ teoriyu pokazatelei Lyapunova [Introduction to the theory of Lyapunov exponents]. Minsk: BGU Publ., 2006, 319 p. ISBN: 985-485-515-5.

13. Lasunskii A.V. Stability and eigenvalues of linear nonautonomous systems of difference and differential equations. Matematika $v$ Vysshem Obrazovanii, 2010, no. 8, pp. 37-40 (in Russian).

Received April 28, 2020

Revised May 16, 2020

Accepted Juny 30, 2020

Alexandr Vasil'evich Lasunskii, Dr. Phys.-Math. Sci., Yaroslav-the-Vise Novgorod State University, Veliky Novgorod, 173003 Russia, e-mail: Alexandr.Lasunsky@novsu.ru.

Cite this article as: A. V. Lasunskii. Refinement of estimates for the Lyapunov exponents of a class of linear nonautonomous systems of difference equations. Trudy Instituta Matematiki i Mekhaniki URO RAN, 2020, vol. 26, no. 3, pp. 84-90. 